\title{
Non-overlapping Domain Decomposition for the Richards Equation via Superposition Operators
}

\author{
Heiko Berninger \\ Fachbereich Mathematik und Informatik, Freie Universität Berlin \\ berninger@math.fu-berlin.de
}

Summary. Simulations of saturated-unsaturated groundwater flow in heterogeneous soil can be carried out by considering non-overlapping domain decomposition problems for the Richards equation in subdomains with homogeneous soil. By the application of different Kirchhoff transformations in the different subdomains local convex minimization problems can be obtained which are coupled via superposition operators on the interface between the subdomains. The purpose of this article is to provide a rigorous mathematical foundation for this reformulation in a weak sense. In particular, this involves an analysis of the Kirchhoff transformation as a superposition operator on Sobolev and trace spaces.

\section{Introduction}

The Richards equation, which describes saturated-unsaturated fluid flow in a homogeneous porous medium, reads

$$
n \theta(p)_{t}-\operatorname{div}\left(K_{h} k r(\theta(p))(\nabla p-z)\right)=0 .
$$

The unknown water or capillary pressure $p$, given as the height of a correponding water column, is a function on $\Omega \times(0, T)$ for a time $T>0$ and a domain $\Omega \subset \mathbb{R}^{d}(d=1,2,3)$ inhibited by the porous medium. $n: \Omega \rightarrow(0,1)$ is the porosity of the soil, $K_{h}: \Omega \rightarrow \mathbb{R}^{+}$is the hydraulic conductivity and $z$ is the coordinate in the direction of gravity.

The saturation $\theta: \mathbb{R} \rightarrow\left[\theta_{m}, \theta_{M}\right]$ with $\theta_{m}, \theta_{M} \in[0,1]$ is an increasing function of $p$ with $\theta(p)=\theta_{M}$ (the case of full saturation and ellipticity of (1)) if $p$ is large enough. The relative permeability $k r:\left[\theta_{m}, \theta_{M}\right] \rightarrow[0,1]$ is an increasing function of $\theta$ with $k r\left(\theta_{M}\right)=1$ which leads to a degeneracy in the elliptic-parabolic equation (1) by $k r\left(\theta_{m}\right)=0$. In this way the Richards equation contains the generalized law of Darcy

$$
\mathbf{v}=-K_{h} k r(\theta(p))(\nabla p-z)
$$




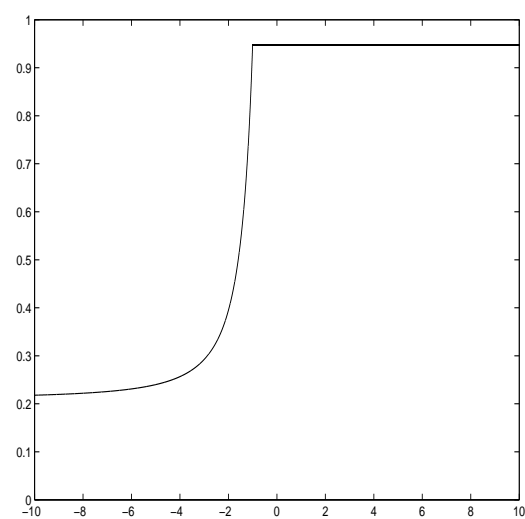

Fig. 1. $p \mapsto \theta(p)$

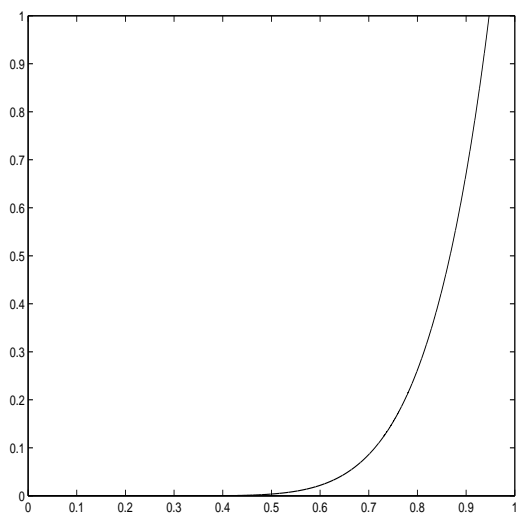

Fig. 2. $\theta \mapsto k r(\theta)$

for the water flux $\mathbf{v}$. Typical shapes of the nonlinearities $\theta$ and $k r$ are depicted in Figures 1 and 2. However, these functions depend on the soil type so that we have different nonlinearities $\theta_{i}, k r_{i}$ on different subdomains $\Omega_{i}$, $i=1, \ldots, k \in \mathbb{N}$, constituting a decomposition of $\Omega$.

In the following we assume $n=K_{h}=1$ and $k=2$ for simplicity. See Figure 3 for a decomposition of $\Omega$ into $\Omega_{1}$ and $\Omega_{2}$ where $\mathbf{n}$ denotes the outer normal of $\Omega_{1}$. Moreover, we assume that (1) is discretized implicitly in time

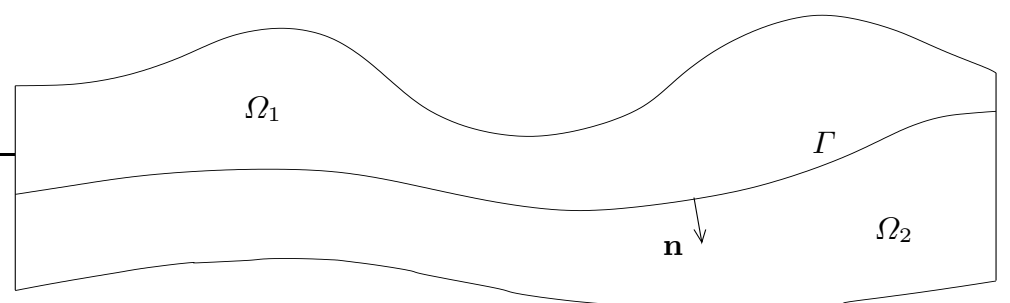

Fig. 3. 2D-domain $\Omega$ decomposed into two subdomains

but with an explicit treatment of the gravitational (convective) term so that with a suitable function $f$ on $\Omega$ we arrive at spatial problems of the form

$$
\theta_{i}\left(p_{i}\right)-\operatorname{div}\left(k r_{i}\left(\theta_{i}\left(p_{i}\right)\right) \nabla p_{i}\right)=f \quad \text { on } \Omega_{i}, \quad i=1,2 .
$$

Appropriate interface conditions on $\Gamma:=\bar{\Omega}_{1} \cap \bar{\Omega}_{2}$, which are motivated hydrologically, are the continuity of the pressure and the normal water flux $\mathbf{v} \cdot \mathbf{n}$ across $\Gamma$. After our implicit-explicit time discretization this leads to

$$
\begin{aligned}
p_{1} & =p_{2} & & \text { on } \Gamma \\
k r_{1}\left(\theta_{1}\left(p_{1}\right)\right) \nabla p_{1} \cdot \mathbf{n} & =k r_{2}\left(\theta_{2}\left(p_{2}\right)\right) \nabla p_{2} \cdot \mathbf{n} & & \text { on } \Gamma .
\end{aligned}
$$


In case of $\theta_{1}=\theta_{2}$ and $k r_{1}=k r_{2}$ these interface conditions can be mathematically derived in a weak sense (and in a very general setting) as a multi-domain formulation for the corresponding global problem, see [3, pp. 131-139] and [11, pp. $5-8]$.

A powerful tool for the treatment of the Richards equation is the Kirchhoff transformation (compare [1] and [4]). Its application eliminates the nonlinearity in front of the spatial derivative in the quasilinear equation (1) so that the transformed equation is semilinear. Here, we need to apply two different Kirchhoff transformations in the two subdomains. More concretely, we define

$$
u_{i}(x):=\kappa_{i}\left(p_{i}(x)\right)=\int_{0}^{p_{i}(x)} k r_{i}\left(\theta_{i}(q)\right) d q \quad \text { a.e. in } \Omega_{i}, \quad i=1,2 .
$$

Consequently, we obtain

$$
k r_{i}\left(\theta_{i}\left(p_{i}\right)\right) \nabla p_{i}=\nabla u_{i}, \quad i=1,2,
$$

by the chain rule so that with the saturation

$$
M_{i}\left(u_{i}\right)=\theta_{i}\left(\kappa_{i}^{-1} u_{i}\right), \quad i=1,2,
$$

with respect to the new variables the equations (2) are transformed into

$$
M_{i}\left(u_{i}\right)-\Delta u_{i}=f \quad \text { on } \Omega_{i}, \quad i=1,2 .
$$

Moreover, the interface conditions read

$$
\begin{aligned}
\kappa_{1}^{-1}\left(u_{1}\right) & =\kappa_{2}^{-1}\left(u_{2}\right) & & \text { on } \Gamma \\
\nabla u_{1} \cdot \mathbf{n} & =\nabla u_{2} \cdot \mathbf{n} & & \text { on } \Gamma
\end{aligned}
$$

in the new variables. Accordingly, boundary conditions on $\partial \Omega$ for (1) and (2) are transformed.

The purpose of the transformation is the following. First, the quasilinear equations (2) are reduced to semilinear equations (8) each containing one nonlinearity $M_{i}$ only. In fact, $M_{i}, i=1,2$, is monotonically increasing such that (with suitable boundary conditions and in a weak formulation) each of the equations (8) is equivalent to a convex minimization problem in a Sobolev space [3, Sec. 2.3]. For appropriate spatial discretizations of such problems monotone multigrid methods provide fast and robust solvers (compare [6] and [3, Sec. 2.6-2.8]). Furthermore, the transformation leads to a continuity condition (10) for the normal fluxes of the transformed variables on the interface $\Gamma$ and infers a nonlinear relationship (9) between these variables induced by superposition operators on $\Gamma$. This enables a convergence analysis for the transmission problem (8)-(10) (with homogeneous Dirichlet boundary conditions on $\Omega$ ) generalizing existing linear theory (see [4] and [3, Sec. 3.3, 3.4]). Note that the Kirchhoff transformation does not necessarily reduce a quasilinear equation as in (2) to a semilinear one if the relative permeability $k r_{i}$ is 
still dependent on $x \in \Omega_{i}$. In this sense homogeneous subproblems referring to the relative permeability $k r$ (not to the hydraulic conductivity $K_{h}(x)$ as in (1)) are required.

In the strong formulations above, the application of Kirchhoff's transformation to the (time-discretized) Richards equation is straightforward. However, with regard to the weak formulations (compare [4, Sec. 3]) some more detailed considerations are necessary in order to prove equivalence of the physical and the Kirchhoff-transformed versions. For example, the chain rule (6) has to be satisfied in a weak sense in $H^{1}\left(\Omega_{i}\right)$. Furthermore, $\kappa_{i}^{-1}\left(u_{i}\right), i=1,2$, in (9) has to be understood as an element of some trace space. In order to clarify these issues, which already occur in the homogeneous case of a single soil in one domain, one has to investigate the Kirchhoff transformations as superposition operators in Sobolev and trace spaces. This is the purpose of this paper.

More concretely, we present weak formulations of the domain decomposition problems for the time-discretized Richards equation as given above, both for the physical and for the Kirchhoff-transformed version in Section 2. Here we also make clear what has to be proved in order to see the equivalence of both weak formulations. Then we carry out some analysis for the Kirchhoff transformation as a superposition operator in Section 3. This leads to positive answers to the open questions raised before and thus to the required equivalence.

\section{Weak forms of the domain decomposition problems and the question of their equivalence}

In this section we give weak formulations of the domain decomposition problems (2)-(4) and (8)-(10) with homogeneous Dirichlet boundary conditions and address the problems we face to prove their equivalence. We start with some necessary notation (compare [4]).

First we abbreviate $k_{i}:=k r_{i} \circ \theta_{i}$ and require $k_{i} \in L^{\infty}(\mathbb{R})$ with $k_{i} \geq \alpha$ for some $\alpha>0$ and $i=1,2$. (For the general case $\alpha=0$ as in Figures 1 and 2 the results are weaker, see [3, Sec. 1.5.4]). Furthermore, let $\theta_{i}, i=1,2$, be bounded Borel-measurable functions on $\mathbb{R}$ and $f \in L^{2}(\Omega), \Omega$ and $\Omega_{i}, i=1,2$, being bounded Lipschitz domains in $\mathbb{R}^{d}$. Now we introduce the spaces

$V_{i}:=\left\{v_{i} \in H^{1}\left(\Omega_{i}\right) \mid v_{i \mid \partial \Omega \cap \partial \Omega_{i}}=0\right\}, \quad V_{i}^{0}:=H_{0}^{1}\left(\Omega_{i}\right), \quad \Lambda:=\left\{v_{\mid \Gamma}: v \in H^{1}(\Omega)\right\}$

and for $w_{i}, v_{i} \in V_{i}$ the forms

$$
a_{i}\left(w_{i}, v_{i}\right):=\left(\nabla w_{i}, \nabla v_{i}\right)_{\Omega_{i}}, \quad b_{i}\left(w_{i}, v_{i}\right):=\left(k_{i}\left(w_{i}\right) \nabla w_{i}, \nabla v_{i}\right)_{\Omega_{i}},
$$

where $(\cdot, \cdot)_{\Omega_{i}}$ stands for the $L^{2}$-inner product on $\Omega_{i}$. The norm in $H^{1}(\Omega)$ will be denoted by $\|\cdot\|_{1}$. Recall that the trace space $\Lambda$ is either $H_{00}^{1 / 2}(\Gamma)$ in case of 
$\Gamma \cap \partial \Omega \neq \emptyset$ (as in Figure 3) or $H^{1 / 2}(\Gamma)$ otherwise [11, p. 7]. The restriction $w_{i \mid \Gamma}$ of a function $w_{i} \in V_{i}$ on the interface $\Gamma$ has to be understood as the application of the corresponding trace operator on $w_{i}$. Finally, let $R_{i}, i=1,2$, be any continuous extension operator from $\Lambda$ to $V_{i}$. Then the variational formulation of problem (2)-(4) with homogeneous Dirichlet boundary conditions reads as follows:

Find $p_{i} \in V_{i}, i=1,2$, such that

$$
\begin{array}{cl}
\left(\theta_{i}\left(p_{i}\right), v_{i}\right)_{\Omega_{i}}+b_{i}\left(p_{i}, v_{i}\right)=\left(f, v_{i}\right)_{\Omega_{i}} & \forall v_{i} \in V_{i}^{0}, i=1,2 \\
p_{1 \mid \Gamma}=p_{2 \mid \Gamma} & \text { in } \Lambda \\
\left(\theta_{1}\left(p_{1}\right), R_{1} \mu\right)_{\Omega_{1}}+b_{1}\left(p_{1}, R_{1} \mu\right)-\left(f, R_{1} \mu\right)_{\Omega_{1}}= & \\
-\left(\theta_{2}\left(p_{2}\right), R_{2} \mu\right)_{\Omega_{2}}-b_{2}\left(p_{2}, R_{2} \mu\right)+\left(f, R_{2} \mu\right)_{\Omega_{2}} \quad \forall \mu \in \Lambda .
\end{array}
$$

Analogously, the weak formulation of the transformed problem (8)-(10) with homogeneous Dirichlet boundary conditions reads:

Find $u_{i} \in V_{i}, i=1,2$, such that

$$
\begin{array}{cl}
\left(M_{i}\left(u_{i}\right), v_{i}\right)_{\Omega_{i}}+a_{i}\left(u_{i}, v_{i}\right)=\left(f, v_{i}\right)_{\Omega_{i}} \quad \forall v_{i} \in V_{i}^{0}, i=1,2 \\
\kappa_{1}^{-1}\left(u_{1 \mid \Gamma}\right)=\kappa_{2}^{-1}\left(u_{2 \mid \Gamma}\right) \quad \text { in } \Lambda \\
\left(M_{1}\left(u_{1}\right), R_{1} \mu\right)_{\Omega_{1}}+a_{1}\left(u_{1}, R_{1} \mu\right)-\left(f, R_{1} \mu\right)_{\Omega_{1}} & \\
-\left(M_{2}\left(u_{2}\right), R_{2} \mu\right)_{\Omega_{2}}-a_{2}\left(u_{2}, R_{2} \mu\right)+\left(f, R_{2} \mu\right)_{\Omega_{2}} \quad \forall \mu \in \Lambda .
\end{array}
$$

Now we can state our main result.

Theorem 1. With the assumptions on $\theta_{i}$ and $k_{i}, i=1,2$, the domain decomposition problem (11)-(13) is equivalent to its Kirchhoff-transformed version (14)-(16).

In the following we point out what is needed to prove Theorem 1 . The arising open questions will be answered positively in Section 3 .

First, since $\theta_{i}$ are bounded Borel-measurable functions on $\mathbb{R}$ we have

$$
\theta_{i}\left(p_{i}(x)\right)=M_{i}\left(u_{i}(x)\right) \quad \text { a.e. on } \Omega_{i}, i=1,2,
$$

due to (7) for all $p_{i} \in V_{i}$ with $u_{i}=\kappa\left(p_{i}\right)$ and the functions given in (17) are Lebesgue-measurable $L^{\infty}$-functions on $\Omega_{i}$.

The two major difficulties in proving Theorem 1 are the following. First, for the equivalence $(11) \Leftrightarrow(14)$ and $(13) \Leftrightarrow(16)$ we need the identity

$$
k_{i}\left(p_{i}\right) \nabla p_{i}=\kappa_{i}^{\prime}\left(p_{i}\right) \nabla p_{i}=\nabla u_{i} \quad \text { a.e. on } \Omega_{i}, \quad i=1,2,
$$

understood as functions in $\left(L^{2}\left(\Omega_{i}\right)\right)^{d}$. This question whether a weak chain rule holds in $H^{1}\left(\Omega_{i}\right)$ can be answered by a very general result (see Theorem 2) which also guarantees 


$$
p_{i} \in H^{1}\left(\Omega_{i}\right) \Longleftrightarrow u_{i} \in H^{1}\left(\Omega_{i}\right) .
$$

Secondly, the equivalence $(12) \Leftrightarrow(15)$ requires the commutativity

$$
\kappa_{i}^{-1}\left(u_{i}\right)_{\mid \Gamma}=\kappa_{i}^{-1}\left(u_{i \mid \Gamma}\right) \quad \text { in } \Lambda, i=1,2,
$$

which turns out to be surprisingly nontrivial, too. Note that this commutativity is also needed to derive the stability of the homogeneous Dirichlet boundary condition

$$
u_{i \mid \partial \Omega \cap \partial \Omega_{i}}=\kappa_{i}\left(p_{i}\right)_{\mid \partial \Omega \cap \partial \Omega_{i}}=\kappa_{i}\left(p_{i \mid \partial \Omega \cap \partial \Omega_{i}}\right)=\kappa_{i}(0)=0, \quad i=1,2,
$$

in a weak sense using (5).

It is clear that the properties (18)-(21) lead to the equivalence in Theorem 1. The purpose of the following section is to make clear that these properties are indeed true.

\section{Kirchhoff transformation as a superposition operator}

It is obvious and has been noted earlier that the equivalence result in Theorem 1 already follows by proving (18)-(21) for a single domain. This shall be done in this section. Therefore, we omit the indices $i \in\{1,2\}$ from now on. In the following we explicitly distinguish the Kirchhoff transformation $\kappa: \mathbb{R} \rightarrow \mathbb{R}$ acting as a function on real numbers $p(x)$ from the transformation which it provides by pointwise (almost everywhere) application on a function $p$ defined (almost everywhere) on $\Omega$.

Definition 1. Let $p$ be a real-valued function defined on a subset $S \subset \mathbb{R}^{d}$, possibly almost everywhere with respect to an appropriate measure. Furthermore, let $\kappa: \mathbb{R} \rightarrow \mathbb{R}$ be a real function. By pointwise application

$$
\left(\kappa_{S}(p)\right)(x):=\kappa(p(x))
$$

of $\kappa$ to $p$ (for $x$ almost everywhere) on $S$ the superposition operator

$$
\kappa_{S}: p \mapsto \kappa(p)
$$

is defined. Let $X$ be a normed space consisting of a subset of all measurable functions on $S$. If the superposition operator satisfies $\kappa_{S}(p) \in X$ for all $p \in X$, we say that it acts on the space $X$. In this case we write

$$
\kappa_{X}: X \rightarrow X
$$

for the restriction of $\kappa_{S}$ on the space $X$ and call $\kappa_{X}$ superposition operator on $X$ (induced by $\kappa$ ). 
For an introduction into the theory of superposition operators (also known as Nemytskij operators) we refer to [2], see also [12]. Here, $S$ will be either $\Omega$ or a submanifold $\Sigma$ of $\partial \Omega$. If not otherwise stated we assume the conditions listed at the beginning of Section 2 and the Kirchhoff transformation $\kappa: \mathbb{R} \rightarrow \mathbb{R}$ given as in (5) with $\kappa(0)=0$ and $\kappa^{\prime}=k$ for the following results. We begin by stating the weak chain rule which goes back to J. Serrin (see [7], [8] and [13]).

Theorem 2. If $\kappa: \mathbb{R} \rightarrow \mathbb{R}$ is Lipschitz continuous the weak chain rule (18) holds for any $p \in W_{\text {loc }}^{1,1}(\Omega)$ provided $\kappa^{\prime}(p(x)) \nabla p(x)$ is interpreted as 0 whenever $\nabla p(x)=0$.

We remark that the last condition is an essential part of the theorem since $\kappa^{\prime}(p(x))$ does not have to be defined for any $x \in \Omega$. Indeed, for $k \in L^{\infty}(\mathbb{R})$ the composition $k \circ p$ alone does not make sense for $p \in W_{\text {loc }}^{1,1}(\Omega)$ since it depends on the choice of the representative in the equivalence class $k$.

The next lemma is not hard to prove (see [3, Sec. 1.5.4]), but of course we must apply the weak chain rule twice in order to obtain (iii).

Lemma 1. The Kirchhoff transformation $\kappa$ has the following properties.

(i) $\kappa: \mathbb{R} \rightarrow \mathbb{R}$ is Lipschitz continuous and has a Lipschitz continuous inverse.

(ii) $\kappa: \mathbb{R} \rightarrow \mathbb{R}$ and $\kappa^{-1}: \mathbb{R} \rightarrow \mathbb{R}$ induce Lipschitz continuous superposition operators acting on $L^{2}(\Omega)$ and on $L^{2}(\Sigma)$ for any submanifold $\Sigma \subset \partial \Omega$.

(iii) $\kappa: \mathbb{R} \rightarrow \mathbb{R}$ induces an invertible superposition operator on $H^{1}(\Omega)$ with

$$
\alpha^{-1}\|p\|_{1} \leq\|\kappa(p)\|_{1} \leq\|k\|_{\infty}\|p\|_{1} \quad \forall p \in H^{1}(\Omega) .
$$

Assertion (iii) now proves (19). Strangely enough, in order to derive the commutativity (20) and (21), respectively, it seems to be necessary to assume the continuity of the superposition operator $\kappa_{H^{1}(\Omega)}$. However, this is a very difficult issue. We just note that by imposing further conditions on the function $k$, e.g. its boundedness and uniform continuity, the continuity of $\kappa_{H^{1}(\Omega)}$ can be proved by elementary means (compare [3, Prop. 1.5.14]) — if one assumes $k$ to be Lipschitz continuous, one even obtains local Lipschitz continuity of $\kappa_{H^{1}(\Omega)}$ in one space dimension (due to Sobolev's embedding theorem).

The following amazing characterization of superposition operators acting on $H^{1}(\Omega)$, however, is a highly nontrivial result from abstract functional analysis. It is due to Marcus and Mizel [9] and [10, pp. 218-220] and essentially states the nonexistence of discontinuous $\kappa_{H^{1}(\Omega)}$.

Theorem 3. Let $\Omega \subset \mathbb{R}^{d}$ be a bounded open set and $\kappa: \mathbb{R} \rightarrow \mathbb{R}$ a Borel function. The superposition operator $\kappa_{\Omega}$ acts on $H^{1}(\Omega)$ if and only if it is continuous on $H^{1}(\Omega)$ or, equivalently, if and only if $\kappa$ is Lipschitz continuous for $d>1$ or locally Lipschitz in the case $d=1$, respectively.

Applying the continuity of the superposition operator $\kappa_{H^{1}(\Omega)}$ we can now prove the commutativity result. In the proof we apply the well-known trace theorem for trace operators $\operatorname{tr}_{\Sigma}: H^{1}(\Omega) \rightarrow H^{1 / 2}(\Sigma)$ (compare e.g. $[5$, pp. $1.61,1.65])$. 
Proposition 1. For a submanifold $\Sigma \subset \partial \Omega$ and $\kappa$ as in Theorem 3 we have the commutativity

$$
\kappa_{\Sigma}\left(\operatorname{tr}_{\Sigma} v\right)=\operatorname{tr}_{\Sigma}\left(\kappa_{\Omega} v\right) \quad \forall v \in H^{1}(\Omega) .
$$

Proof. We prove that for any $v \in H^{1}(\Omega)$

$$
\left\|\operatorname{tr}_{\Sigma}\left(\kappa_{\Omega} v\right)-\kappa_{\Sigma}\left(\operatorname{tr}_{\Sigma} v\right)\right\|_{L^{2}(\Omega)}
$$

is arbitrarily small by considering a sequence $\left(v_{n}\right)_{n \in \mathbb{N}} \subset C^{\infty}(\bar{\Omega})$ converging to $v$ in $H^{1}(\Omega)$. In fact, since Theorem 3 provides the continuity of $\kappa$ and the trace of a continuous function on $\Sigma$ coincides with its restriction to $\Sigma$, the norm in (23) can be estimated by

$$
\left\|\operatorname{tr}_{\Sigma}\left(\kappa_{\Omega} v\right)-\left(\kappa_{\Omega} v_{n}\right)_{\mid \Sigma}\right\|_{L^{2}(\Omega)}+\left\|\kappa_{\Sigma}\left(v_{n \mid \Sigma}\right)-\kappa_{\Sigma}\left(t r_{\Sigma} v\right)\right\|_{L^{2}(\Omega)} .
$$

The first term in (24) is at most

$$
\left\|t r_{\Sigma}\right\|\left\|\kappa_{\Omega} v-\kappa_{\Omega} v_{n}\right\|_{1}
$$

due to the trace theorem, and this estimate goes to 0 for $n \rightarrow \infty$ by the continuity of $\kappa_{H^{1}(\Omega)}$. For $d>1$ where $\kappa: \mathbb{R} \rightarrow \mathbb{R}$ is Lipschitz continuous the second term in (24) can be estimated by

$$
L\left(\kappa_{L^{2}(\Sigma)}\right)\left\|v_{n \mid \Sigma}-\operatorname{tr}_{\Sigma} v\right\|_{L^{2}(\Sigma)} \leq L\left(\kappa_{L^{2}(\Sigma)}\right)\left\|t r_{\Sigma}\right\|\left\|v_{n}-v\right\|_{1}
$$

with Lemma 1 (ii) $\left(L\left(\kappa_{L^{2}(\Sigma)}\right)\right.$ denotes the Lipschitz constant of $\left.\kappa_{L^{2}(\Sigma)}\right)$ and the trace theorem and, therefore, tends to 0 for $n \rightarrow \infty$, too. In one space dimension (22) is clear due to Sobolev's embedding theorem ( $v$ is continuous) and the trace theorem.

Note that although Proposition 1 guarantees the commutativity in (20), we do not yet know whether this indeed takes place in the trace space $\Lambda$, i.e. if we have $\kappa_{i}^{-1}\left(u_{i \mid \Gamma}\right) \in \Lambda$. In the following we do not only prove this acting condition but also the continuity of the Kirchhoff transformation and its inverse as superposition operators on trace spaces.

Proposition 2. For a submanifold $\Sigma \subset \partial \Omega$ the function $\kappa$ as in Theorem 3 induces a continuous superposition operator on $H^{1 / 2}(\Sigma)$ and, if $\kappa(0)=(0)$, on $H_{00}^{1 / 2}(\Sigma)$, too.

Proof. With the continuous extension operator $R_{\Sigma}: H^{1 / 2}(\Sigma) \rightarrow H^{1}(\Omega)$ given by the trace theorem and using Proposition 1 we can write

$$
\kappa_{\Sigma}=\kappa_{\Sigma} \circ t r_{\Sigma} \circ R_{\Sigma}=t r_{\Sigma} \circ \kappa_{H^{1}(\Omega)} \circ R_{\Sigma}
$$

and the operator on the right hand side is a composition of continuous operators which obviously acts on $H^{1 / 2}(\Sigma)$. 
Regarding the second case we recall (see $\left[5\right.$, p. 1.60]) that $H_{00}^{1 / 2}(\Sigma)$ is the space of all functions $v \in H^{1 / 2}(\Sigma)$ allowing trivial extensions $\tilde{v} \in H^{1 / 2}(\partial \Omega)$ with the norm

$$
\|v\|_{H_{00}^{1 / 2}(\Sigma)}=\|\tilde{v}\|_{H^{1 / 2}(\partial \Omega)} .
$$

Now, let $\eta \in H_{00}^{1 / 2}(\Sigma)$ and $\tilde{\eta}$ be a trivial extension of $\eta$ in $H^{1 / 2}(\partial \Omega)$. Then, since $\kappa(0)=0$ and $\kappa_{\partial \Omega}$ acts on the space $H^{1 / 2}(\partial \Omega)$, we can conclude $\kappa_{\partial \Omega}(\tilde{\eta}) \in H^{1 / 2}(\partial \Omega)$ and $\kappa_{\partial \Omega}(\tilde{\eta})_{\mid \Sigma}$ is a trivial extension of $\kappa_{\Sigma}(\eta) \in H^{1 / 2}(\Sigma)$, i.e. by definition $\kappa_{\Sigma}(\eta) \in H_{00}^{1 / 2}(\Sigma)$. Moreover, if $\mu \in H_{00}^{1 / 2}(\Sigma)$ is treated as $\eta$, then $\kappa_{\partial \Omega}(\tilde{\eta})-\kappa_{\partial \Omega}(\tilde{\mu}) \in H^{1 / 2}(\partial \Omega)$ is a trivial extension of $\kappa_{\Sigma}(\eta)-\kappa_{\Sigma}(\mu) \in$ $H_{00}^{1 / 2}(\Sigma)$. Now, $(25)$ and the continuity of $\kappa_{\partial \Omega}$ provide that for any $\varepsilon>0$ we have

$$
\left\|\kappa_{\Sigma}(\eta)-\kappa_{\Sigma}(\mu)\right\|_{H_{00}^{1 / 2}(\Sigma)}=\left\|\kappa_{\partial \Omega}(\tilde{\eta})-\kappa_{\partial \Omega}(\tilde{\mu})\right\|_{H^{1 / 2}(\partial \Omega)} \leq \varepsilon
$$

if $\|\tilde{\eta}-\tilde{\mu}\|_{H^{1 / 2}(\partial \Omega)}=\|\eta-\mu\|_{H_{00}^{1 / 2}(\Sigma)} \leq \delta$ holds with a suitable $\delta>0$.

For completeness we remark that Proposition 2 also holds for the trace space $H_{0}^{1 / 2}(\Sigma)$, see [3, Prop. 1.5.17].

We close this investigation by noting that, in addition to Dirichlet and Neumann boundary conditions, which have been considered above, boundary conditions of "Signorini-type" can also be suitably Kirchhoff-transformed in a weak sense. However, as in the degenerate case of $\alpha=0$ one can no longer establish the full equivalence result, compare [3, Thm. 1.5.18].

\section{References}

[1] H.W. Alt and S. Luckhaus. Quasilinear elliptic-parabolic differential equations. Math. Z., 183:311-341, 1983.

[2] J. Appell and P.P. Zabrejko. Nonlinear superposition operators. Cambridge University Press, 1990.

[3] H. Berninger. Domain Decomposition Methods for Elliptic Problems with Jumping Nonlinearities and Application to the Richards Equation. PhD thesis, Freie Universität Berlin, 2007.

[4] H. Berninger, R. Kornhuber, and O. Sander. On nonlinear DirichletNeumann algorithms for jumping nonlinearities. In O.B. Widlund and D.E. Keyes, editors, Domain Decomposition Methods in Science and Engineering XVI, volume 55 of LNCSE, pages 483-490. Springer, 2007.

[5] F. Brezzi and G. Gilardi. Functional spaces. In H. Kardestuncer and D.H. Norrie, editors, Finite Element Handbook, chapter 2 (part 1), pages 1.29-1.75. Springer, 1987.

[6] R. Kornhuber. On constrained Newton linearization and multigrid for variational inequalities. Numer. Math., 91:699-721, 2002. 
[7] G. Leoni and M. Morini. Necessary and sufficient conditions for the chain rule in $W_{\text {loc }}^{1,1}\left(\mathbb{R}^{N} ; \mathbb{R}^{d}\right)$ and $B V_{\text {loc }}\left(\mathbb{R}^{N} ; \mathbb{R}^{d}\right)$. J. Eur. Math. Soc. (JEMS), 9 (2):219-252, 2007.

[8] M. Marcus and V.J. Mizel. Absolute continuity on tracks and mappings of sobolev spaces. Arch. rat. Mech. Anal., 45:294-320, 1972.

[9] M. Marcus and V.J. Mizel. Complete characterization of functions which act, via superposition, on Sobolev spaces. Trans. Amer. Math. Soc., 251: 187-218, 1979.

[10] M. Marcus and V.J. Mizel. Every superposition operator mapping one Sobolev space into another is continuous. J. Funct. Anal., 33:217-229, 1979.

[11] A. Quarteroni and A. Valli. Domain Decomposition Methods for Partial Differential Equations. Oxford Science Publications, 1999.

[12] T. Runst and W. Sickel. Sobolev Spaces of Fractional Order, Nemytskij Operators, and Nonlinear Partial Differential Equations. De Gruyter, 1996.

[13] J. Serrin and D. Varberg. A general chain rule for derivatives and the change of variables formula for the Lebesgue integral. Am. Math. Mon., pages $514-520,1969$. 